\title{
How yarn orientation limits fibrotic tissue ingrowth in a woven polyester heart valve scaffold: a case report
}

https://doi.org/10.1515/bmt-2020-0137

Received May 22, 2020; accepted July 16, 2020; published online August 3, 2020

\begin{abstract}
Transcatheter Aortic Valve Implantation (TAVI) has become today a popular alternative technique to surgical valve replacement for critical patients. However, with only six years follow up on average, little is known about the long-term durability of transcatheter implanted biological tissue. Moreover, the high cost of tissue harvesting and chemical treatment procedures favor the development of alternative synthetic valve leaflet materials. In that context, thin, strong and flexible woven fibrous constructions could be considered as interesting candidates. However, the interaction of textile material with living tissue should be comparable to biological tissue, and the Foreign Body Reaction (FBR) in particular should be controlled. Actually, the porosity of textile materials tends to induce exaggerated tissue ingrowth which may prevent the implants from remaining flexible. The purpose of this preliminary animal case study is to investigate the influence of the valve leaflet yarn orientation on the fibrotic tissue ingrowth. For that purpose the in vivo performances of $45^{\circ}$ inclined yarn woven valve leaflets implanted in juvenile sheep model were assessed after three months implantation. Results bring out that in the frame of this case study the development of fibrosis is limited with a woven fabric valve obtained from $45^{\circ}$ inclined yarns.
\end{abstract}

Keywords: foreign body reaction; medical textiles; textile biomaterials; textile heart valve.

Since 2002 and Cribier's first success in using the transcatheter technique to replace a faulty aortic valve

*Corresponding author: Frederic Heim, Laboratoire de Physique et Mécanique Textiles (LPMT), ENSISA, 68093, Mulhouse, France,

E-mail: frederic.heim@uha.fr

Anne Meddahi-Pelle and Graciela Pavon-Djavid, INSERM U1148, Laboratory for Vascular Translational Science, Université Sorbonne Paris Nord, Villetaneuse, France

Nabil Chakfe, GEPROVAS, Hôpitaux Universitaires de Strasbourg, Strasbourg, France
Transcatheter Aortic Valve Implantation (TAVI), minimally invasive valve replacement has been performed successfully over 300,000 times in western countries [1]. A closer look to recent market forecasts shows that the market share of this technology is expected to grow by $14 \%$ a year up to 2025. One may assume that the technology becoming more mature, it could be applied to less critical patient in a near future. But with longer life expectation for the patient, the long term durability of the valve material must be guaranteed.

Transcatheter devices implanted clinically today use porcine or bovine tissue as valve leaflet material. In some cases, these materials have shown to be durable up to 10 years [2]. However, when observed at larger scale it comes out that once assembled inside the metallic stent and crimped at low diameter for catheter insertion, the biological material undergoes additional stress and gets degraded already before implantation [3-5]. A first long term durability study about TAVI first generation devices has been recently presented and shows high rates of valve degeneration after a few years [6]. Biological material, which proved long term durability when mounted on a rigid stent and implanted through open chest surgery, appears to be potentially fragile on the long term at different levels for catheter implantation [7, 8].

In order to address the lack of long term experience with biological tissue, it is relevant to investigate the use of synthetic materials for leaflet replacement. Synthetic valves would avoid the cost of biological tissue harvesting and preservation. In that context, textile material could be considered as a potential alternative to replace biological valve leaflets. This material presents unique strengths and folding properties and can be obtained in various configurations: non-woven, woven or knitted. Several research projects have focused over the last years on investigating the potential of non-woven constructions as fibrous scaffolds for valve tissue engineering [9-11]. But no convincing results regarding the long term durability were obtained.

With respect to woven textile, several studies have been published in the last years about the in vitro and in 
vivo behavior of polyester woven valve leaflets [12, 13]. Textile and biological tissue were compared in terms of mechanical and hydrodynamic performances and results show that the behavior of textile is close to the behavior of biological tissue [14]. Vaesken et al. implanted successfully woven valve prototypes made from various textile constructions in sheep models over a six months period of time. Authors show that the standard foreign body reaction (FBR), which is characterized by encapsulation of the synthetic leaflets with fibrotic tissue, is largely influenced by the topography of the leaflet surface [15]. It comes out in particular that valves obtained from woven fabric manufactured with multifilament yarns oriented parallel to the valve axis, which appear to be the most durable fibrous constructions in vitro, undergo significant tissue ingrowth in vivo. In another study by the same group it had been shown that a fibrosis layer is formed on multifilament valve leaflets already two months after implantation in a sheep model [16]. In order to limit fibrotic tissue ingrowth, one strategy would consist in increasing the deformability of the fibrous substrate. Inclining the yarns in the leaflet by $45^{\circ}$ to the valve axis, would allow making the leaflet more deformable. The purpose of this preliminary work was to assess, in the frame of a case study, the effect of the yarn orientation on the FBR occurring in vivo in a valve implanted in mitral position in a sheep model over a three months period of time.

Two valves were obtained from a micro denier fabric tubular membrane (Cardial, St Etienne, France) shape set and thermo-fixed to get a semi-lunar geometry for the valve cusps as was already described in a previous work [17]. The fabric membrane was however oriented at $45^{\circ}$ before generating the tube in order to make the yarns running inclined rather than parallel with regard to the tube axis. The textile was a woven polyester construction (multifilament, 75 yarns $/ \mathrm{cm}$ in warp direction, 50 yarns $/ \mathrm{cm}$ in weft direction, $75 \mathrm{~g} / \mathrm{m}^{2}$, 50 dtex yarns). For each valve specimen the fabric membrane was sutured with an Ethicon monofilament suture yarn (Prolene 5-0) on a $23 \mathrm{~mm}$ Sorin stent of a Mitroflow bioprosthesis (Sorin, Milano, Italy) from which the biological valve was removed and replaced by the textile valve. In this assembling procedure the original suture cuff was kept on the stent. The valve specimens were prepared and sterilized with Ethylene Oxide (EtO).

After cardiopulmonary bypass, the prototypes were implanted in mitral position in juvenile sheep models (Laboratoire de Recherches Biochirurgicales de la Foundation Alain Carpentier, Paris). In this preliminary study, two sheeps weighing $40 \mathrm{~kg}$ on average were used as animal models. Mitral position was preferred to aortic position to limit the risks of the surgical procedure, while respecting,

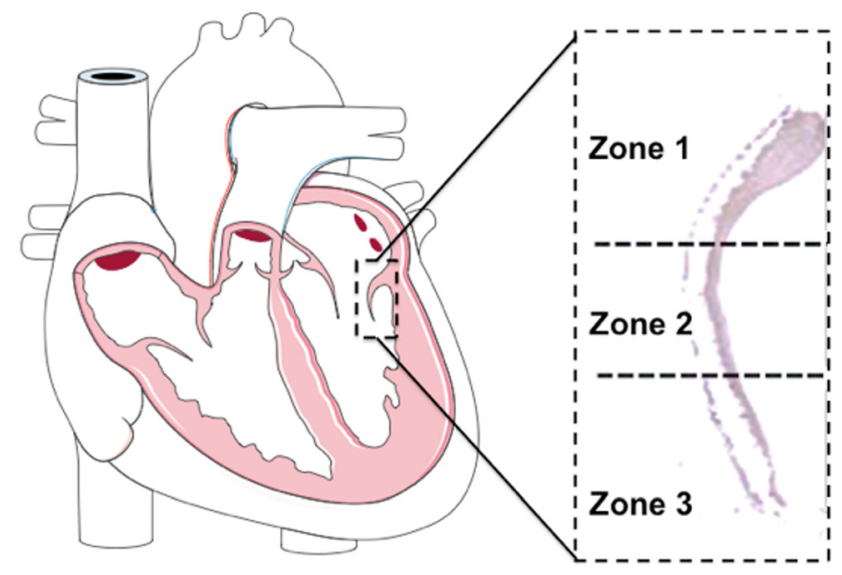

Figure 1: Valve leaflet zones considered for pannus thickness measurement (reproduced with permission from ref. [15]).

however, the physiological pressure conditions. Experimental procedure was performed in accordance with the French regulation (agreement number MESR N ${ }^{\circ} 01425.04$ ) after the agreement of the local animal ethic committee. One animal died the day of procedure. While the valve was working properly at the echo, a malfunction of the left ventricle was detected (supposed to be due to brain or coronary embolism) and was not compatible with the animal survival. The second animal was kept alive for three months before being sacrificed. The valve was then explanted and was analyzed at histological level in order to observe the fibrotic capsule on the leaflets.

After macroscopic examination, implanted materials were dissected and at least $0.5 \mathrm{~cm}$ of surrounding tissue was excised and gently rinsed in saline before utilization. For histological analysis, samples were fixed in a $4 \%$ paraformaldehyde solution, dehydrated, and embedded in

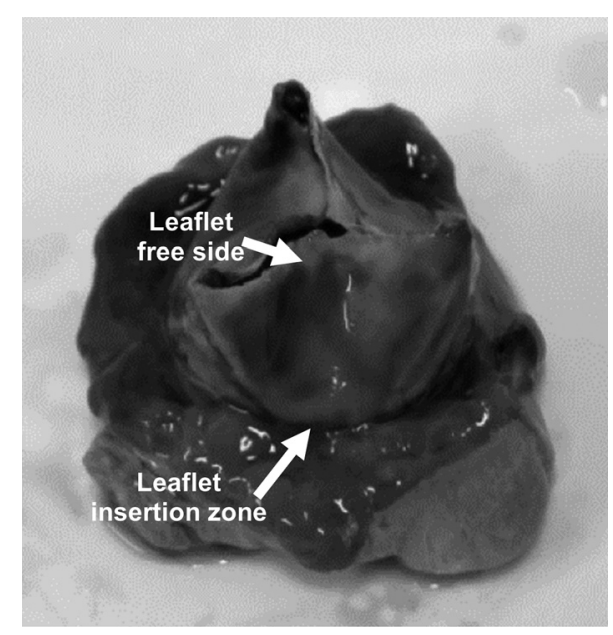

Figure 2: Valve explant. 

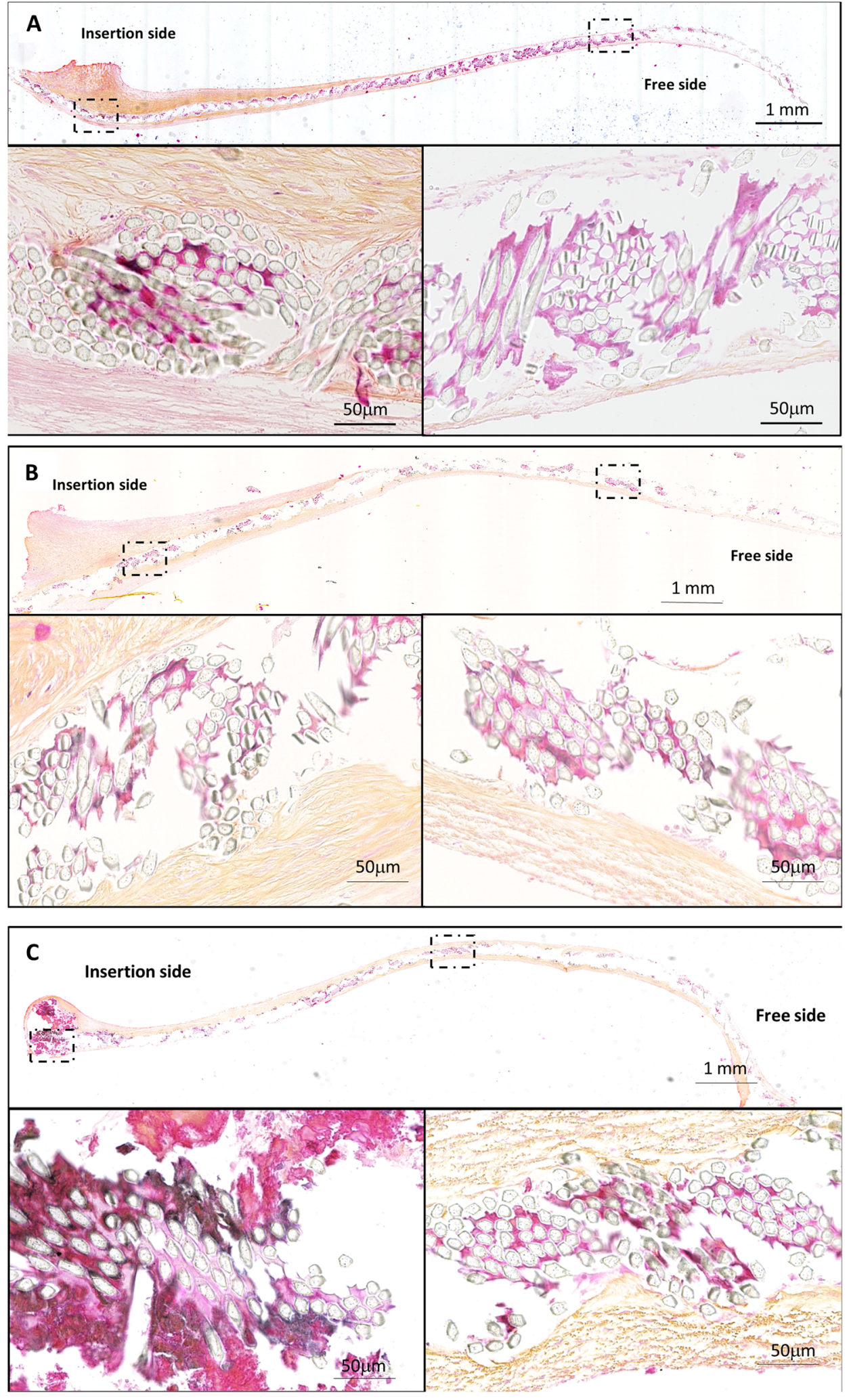

Figure 3: Histological analysis (Masson Trichrome). Masson Trichrome-stained cross sections of the three explanted leaflets: (A), (B) and (C). Cell nuclei are seen in blue/black, cell cytoplasm's in pink and elastic fibers in red. Macroscopic sections and magnifications show that the tissue (pink/red) surrounds the fibers (white). 


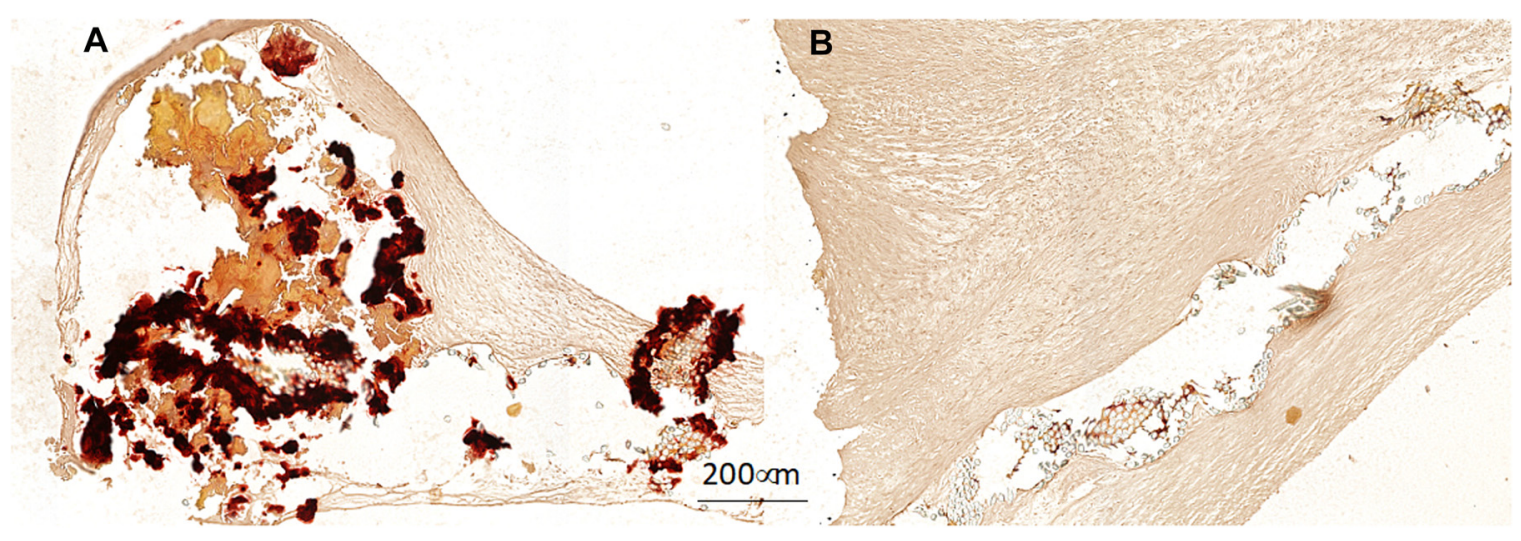

Figure 4: Histological analysis (Alizarin Red). Alizarin Red-stained cross section for calcification detection. The presence of calcium deposits (hydroxy-apatite) is observed in dark brown color (A): leaflet insertion zone, (B): leaflet free zone.

paraffin. Eight-micron-thick serial sections were obtained (Leitz Wetzlar microtome, France). Nuclear, cytoplasmic and collagen staining were evidenced on sections by a Masson's trichrome stain. Digital sections were acquired with a NanoZoomer 2.0-RC C 10730 (Hamamatsu, Japan).

In order to determine the fibrous capsule thickness three different areas were determined and analyzed on each digital section: (1) leaflet insertion zone to the aortic wall, (2) leaflet central zone, (3) leaflet free side (Figure 1). A quantitative analysis was performed by calculating an average fibrotic tissue thickness from values regularly measured along each zone length $(n=5)$ Finally, considering three leaflet sections for the valve, 15 values could thus be considered to assess the average fibrosis thickness in each zone. In addition, Alizarin red staining was used for calcification detection analysis.

The explanted valve can be observed at macroscopic level in Figure 2. The textile leaflet edge can still be clearly identified as the fibrous pannus thickness is not exaggerated. Figure 3 shows cross sections of the explanted valve leaflets at histological level (paraffin section). In the figure fibrotic tissue appears in pink color while the fabric filaments can be identified in between in white color. Results

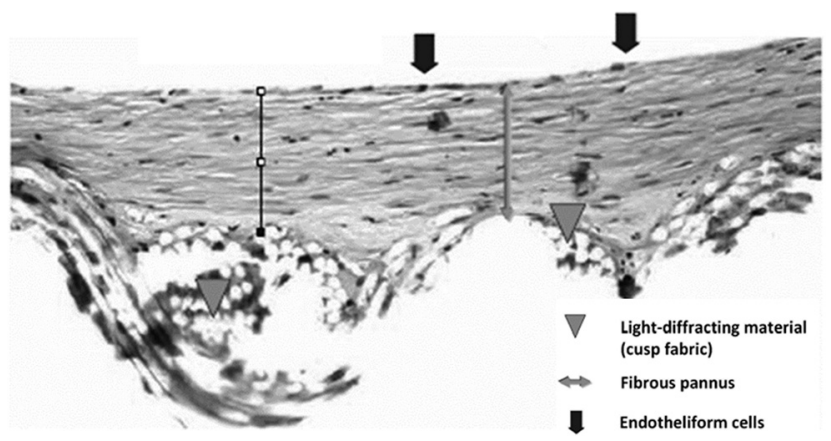

Figure 5: Pannus at the insertion zone in the straight yarn valve. confirm that the cusp fabric material was only slightly covered by a continuous thin fibrous pannus which enlarges at its insertion end (ring/valve assembling zone). The observation was similar for all three valve leaflets. From a quantitative point of view, the average thickness of the fibrotic layer was $182 \mu \mathrm{m} \pm 105$ in zone 1 (leaflet insertion zone), $50 \mu \mathrm{m} \pm 23$ in zone 2 and $42 \mu \mathrm{m} \pm 21$ in zone 3 (leaflet free end).

Figure 4 is a typical view of how calcification occurs between filaments in a fabric (dark zones)

This case study included one animal, which was explanted at three months follow-up. For that reason the interpretation of the results cannot give a statistically significant assessment. However, several comments can be made which give an early comprehensive description of the results.

A first observation is that the fibrotic proliferation was globally limited whatever the considered sample leaflet. Out of the insertion zone, only a smooth tissue layer could be observed on the leaflets from the middle of the leaflet towards its free end. Regarding the insertion area the pannus thickness was larger in that zone as the valve is fixed to the ring and the ring to the aortic root. Fibrotic tissue formation is initiated in that wounded zone first. Proliferation is easier in that zone compared to the leaflet length which is more mobile. This is consistent with the fact that fibroblasts develop better on more static and rigid substrates.

However, when comparing the results obtained here with the results published in a previous paper in which the same fabric valve, however straight oriented (yarns running parallel to the valve axis), was implanted two months in a sheep model, significant differences appear [16]. Actually, in spite of a shorter implantation time (60 vs. 90 days in the present work), the average pannus thickness in the straight yarn valve was nearly two times 


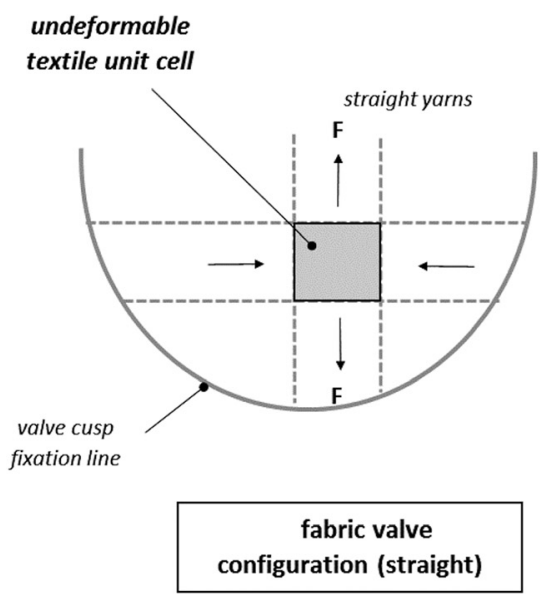

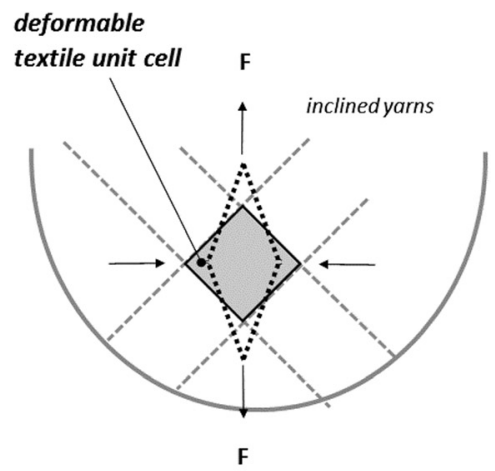

fabric valve configuration $\left(45^{\circ}\right)$
Figure 6: Fabric unit cell deformation mechanism at $45^{\circ}$ orientation. thicker at the insertion zone (330 vs $182 \mu \mathrm{m}$ ) while zone 2 and zone 3 had not been considered in that previous work. Figure 5 shows the pannus at the insertion zone obtained with the straight yarn valve. In that case, the pannus limited largely the mobility of the leaflets and led to the premature sacrifice of the animal already after 60 days. In the present work, the valve leaflets were still mobile after three months even if some calcifications could be identified withing the fibrous assembly, inducing local rigidifications. This result seems to demonstrate that the yarn orientation influences the FBR. Actually, woven textile (as used here) is structurally completely anisotropic. The material is nearly nondeformable when extended in warp and weft directions, but deformable at $45^{\circ}$ as yarns can rotate over each other as described in Figure 6. In the particular configuration of the valve, as one edge of the textile is free (leaflet edge), inclining the fabric at $45^{\circ}$ induces more deformability of the textile (compared to straight). Basically, when the valve is in closed position, during the heart diastolic phase, the pressure applied on the leaflets can go up to $140 \mathrm{mmHg}$. This pressure induces a $1.8 \mathrm{MPa}$ stress in the $0.1 \mathrm{~mm}$ thick leaflet membrane, which corresponds to a 3.6 $\mathrm{N}$ extension force (force $\mathrm{F}$ in Figure 6) applied along the free edge of the leaflet. When the textile is inclined, this extension force overcomes the friction forces occurring between the textile yarns which rotate relatively to each other. This leads to fabric deformation. This deformation mechanism can limit the proliferation of fibroblasts, which tend to develop less on mobile substrates, as the stiffness of those is reduced.

With respect to the calcification phenomenon, Figure 4 demonstrates that calcium crystals tend to grow in some areas located between filaments (dark colored zones in the figure). Actually, a multifilament assembling is characterized by contact zones between filaments which can be considered as nucleation sites promoting calcium fixation. This creates local rigidification areas in the textile, which in the long term would limit the leaflet mobility. This remains a critical concern which is specific to woven fibrous scaffolds whatever the yarn orientation. This issue needs to be addressed in future work with physicochemical treatments like plasma surface functionalization for instance.

The fact that only one animal was considered within the frame of this case report, limits the interpretation of the result obtained. More animals need to be involved in a future more global pre-clinical study in order to better assess the effective potential of woven fibrous constructions as valve leaflet material.

The early results which can be observed in the frame of this case study support that a woven textile scaffold implanted in vivo in mitral position undergoes limited fibrin deposition if the yarns are oriented $45^{\circ}$ from the valve axis. Thin fibrous pannus develops mainly on the outflow side of the valve cusps. The thickness value of the biological layer observed in the frame of this case study is $50 \%$ lower than the one obtained when the yarns are straight. The observation made supports preliminarily the assumption that the deformability of the fibrous construction induced by the inclined yarns tends to limit the fibrotic tissue development in the frame of this case study. But more systematic trials are now necessary to be done to confirm that trend.

Research funding: None declared.

Author contributions: All authors have accepted responsibility for the entire content of this manuscript and approved its submission.

Competing interests: Authors state no conflict of interest. Informed consent: Informed consent was obtained from all individuals included in this study. 
Ethical approval: Experimental procedure was performed in accordance with the french regulation (agreement number MESR $\left.\mathrm{N}^{\circ} 01425.04\right)$ after the agreement of the local animal ethic committee.

\section{References}

1. O'Sullivan CJ, Wenaweser P. A glimpse into the future: in 2020 , which patients will undergo TAVI or SAVR? Intervent Cardiol 2017; 24:44-50.

2. Sathananthan J, Lauck S, Polderman J, Yu M, Stephenson A, Sathananthan G, et al. Ten year follow-up of patients treated with transcatheter aortic valve implantation. Paris, France: Euro PCR 2019; 2019.

3. Zegdi R, Ciobotaru V, Noghin M, Sleilaty G, Lafont A, Latrémouille $C$, et al. Is it reasonable to treat all calcified stenotic aortic valves with a valved stent. J Am Coll Cardiol 2008;51:579-84.

4. Kiefer P, Gruenwald F, Kempfert J, Aupperle H, Seeburger J, Mohr FW, et al. Crimping may affect the durability of transcatheter valves: an experimental analysis. Ann Thorac Surg 2011;92:155-60.

5. Alavi H, Groves E, Kheradvar A. The effects of transcatheter valve crimping on pericardial leaflets. Ann Thorac Surg 2014;97:1260-6.

6. Dvir D. Half of transcatheter heart valves show degeneration within 10 years of TAVI. Paris, France: EuroPCR; 2016.

7. van Nooten G, Ozaki S, Herijgers P, Segers P, Verdonck P, Flameng $W$. Distortion of the stentless porcine valve induces accelerated leaflet fibrosis and calcification in juvenile sheep. J Heart Valve Dis 1999;8:34-41.

8. Cheung AW, Gurvitch R, Ye J, Wood D, Lichtenstein SV, Thompson $C$, et al. Transcatheter transapical mitral valve-in-valve implantations for a failed bioprosthesis: a case series. J Thorac Cardiovasc Surg 2011;141(3):711-5.
9. Haller N, Hollweck T, Thierfelder N, Schulte J, Hausherr JM, Dauner $M$. Noninvasive analysis of synthetic and decellularized scaffolds for heart valve tissue engineering. Am Soc Artif Intern Organs J 2013;59:169-77.

10. Kucinska-Lipka J, Gubanska I, Janik H., Sienkiewicz M. Fabrication of polyurethane and polyurethane based composite fibers by the electrospinning technique for soft tissue engineering of cardiovascular system. Mater Sci Eng C 2015;46:166-76.

11. Amoroso NJ, D'Amore A, Hong Y, Rivera CP, Sacks MS, Wagner WR. Microstructural manipulation of electrospun scaffolds for specific bending stiffness for heart valve tissue engineering. Acta Biomater 2012;8:4268-77.

12. Vaesken A, Heim F, Chakfe N. Fiber heart valve prosthesis: influence of the fabric construction parameters on the valve fatigue performances. J Mech Behav Biomed 2014;40: 69-74.

13. Heim F, Durand B, Chakfe N. Textile heartvalve prosthesis: manufacturing process and prototype performances. Textil Res J 2008;78:1124-31.

14. Yousefi A, Vaesken A, Amri A, Dasi LP, Heim F. Heart valves from polyester fibers vs. biological tissue: comparative study in vitro. Ann Biomed Eng 2017;45:476-86.

15. Vaesken A, Pelle A, Pavon-Djavid G, Rancic J, Chakfe N, Heim F. Heart valves from polyester fibers: a preliminary 6-month in vivo study. Biomed Eng Biomed Tech 2018;6:271-8.

16. Heim F, Durand B, Chafke N. Textile heart valve prosthesis: from fabric design criteria to early in-vivo performances. J Heart Valve Dis 2013;22:361-7.

17. Heim F, Durand B, Chakfe N. Textile heart valve: novel shaping process and material performances. Mater Manufact Proces 2011; 26:1303-9. 